\title{
Space videos on YouTube - what makes the audience tick
}

\author{
Maarten Roos ${ }^{1,}{ }^{*}$ Jan Van den Bulck ${ }^{2}$ \\ ${ }^{1}$ Lightcurve Films, Portugal. \\ 2University of Michigan, Dept. of Communication Studies, USA.
}

\begin{abstract}
Nowadays it is easier than ever before to produce films and videos and make them available to a worldwide audience via platforms such as YouTube, Twitter and Facebook, among others. The European Space Agency (ESA), the European Southern Observatory (ESO), the National Aeronautics and Space Administration (NASA), and other similar organisations constantly produce videos aimed at the general interested audience, and distribute them on through their social media channels. Different formats are offered such as educational, informative, news style, science stories, scientist profiles, behind-the-scenes, animations and data based animations. But which of these formats do really stick and why? A simple statistical analysis of 106 videos found on the ESA, ESO and NASA YouTube channels shows that videos based on animations and the representation of data, with little to no explanation and accompanied by music are the more popular in terms of views per month by about a factor of two compared to other types of videos. This can likely be explained by the higher entertainment value of such videos.
\end{abstract}

\section{Rationale}

The production of videos with scientific content in general, and space related content in particular, has seen an enormous rise over the past decade or so. This can be attributed to the significant lowering of overall video production costs and the ease of worldwide distribution through internet social networks. Space organisations such as the European Space Agency (ESA) [1] and the European Southern Observatory (ESO) [2] have dedicated YouTube channels, whereas the National Aeronautics and Space Administration (NASA) has several [3], and together they contain many thousands of space related videos.

In 2013 the first author of this paper, Maarten Roos (MR), applied for a position as Video Producer for Mars and Planetary Science at the communication team of the NASA Goddard Space Flight Center (GSFC). As a filmmaker with relevant skills and portfolio and a strong planetary science background, he made it to the short list and the (telephone) interview. As part of MR's preparation for the interview, he not only looked at the content that the GSFC communication team was putting out on their YouTube channel [3], but also at how popular 
that content actually is. He did a simple statistical analysis on a sample of 49 videos from the GSFC NASA Explorer YouTube channel. When presenting the results during the interview, MR was surprised to learn that the people at the GSFC communication team had not done such an analysis for themselves.

In what follows we present a more extended version of this initial analysis, including content from the ESA and ESO YouTube channels.

\section{The sample and simple statistics}

Initially, MR selected 49 videos exclusively from the GSFC NASA Explorer YouTube channel [3]. After watching each video, it was classified in one of five categories:

(1) Animations and Data (13 videos);

(2) Science Stories (6 videos);

(3) Educational (15 videos);

(4) Documentary, Informative and News Stories (7 videos);

(5) People Profiles (8 videos).

The date of publication and the total number of views were recorded, which is available for any YouTube video on the video's page. The only selection criteria was that the video had to be online more than 180 days before noting down the statistical data. This was to make sure that enough time had passed between the date of publication and the date of observation, i.e. the moment that the video data was logged. Observing that criterion a meaningful average of the views per month could be calculated, as explained below. The exercise was first done on 15 April 2013 and redone four years later, on 21 June 2017. In Table 1 all this data from the NASA Explorer YouTube channel is presented, including the links to each video as part of the title.

For each video the average views per month were calculated from the number of views on the day of recording and the number of days between the day of recording and the day of publication, taking a 30-day month.

For each of the five categories the median value of the average views per month of all the videos in that category was calculated. The median value as opposed to the average was used, because the median is less sensitive to outliers for the small samples that we have here.

In the next step, videos from the ESA and ESO YouTube channels were added, using the same method and criteria, for 21 June 2017. The final sample contains a total of 106 videos, distributed among the categories as follows:

(1) Animations and Data (30 videos);

(2) Science Stories (14 videos);

(3) Educational (32 videos);

(4) Documentary, Informative and News Stories (21 videos);

(5) People Profiles (9 videos).

The full data for 21 June 2017 is presented in Table 2. 


\section{What could it mean?}

\subsection{The big picture}

In Figure 1 the bar diagram of the median values for all the categories for the NASA Explorer YouTube channel is shown for 15 April 2013 and in Figure 2 the same data is shown for 21 June 2017 (Table 1).

\section{NASA GSFC YOUTUBE CHANNEL; 49 VIDEOS; 15-04-2013}

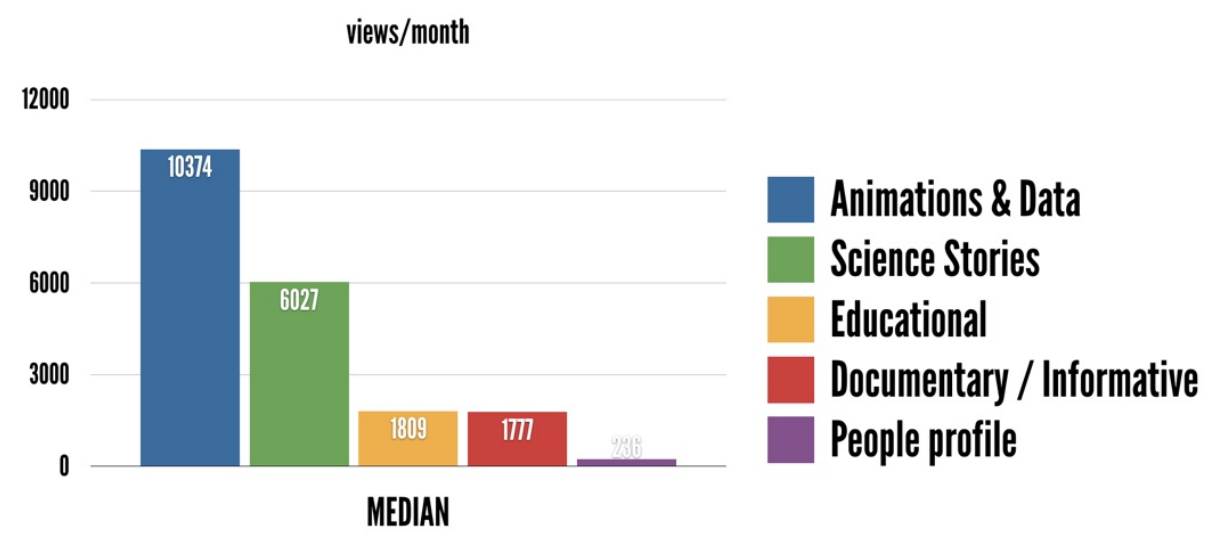

Fig. 1. The median value of the views per month for each category in the initial selection of 49 video of the NASA Explorer YouTube channel on 15 April 2013. 


\section{NASA GSFC YOUTUBE CHANNEL; 49 VIDEOS; 21-06-2017}

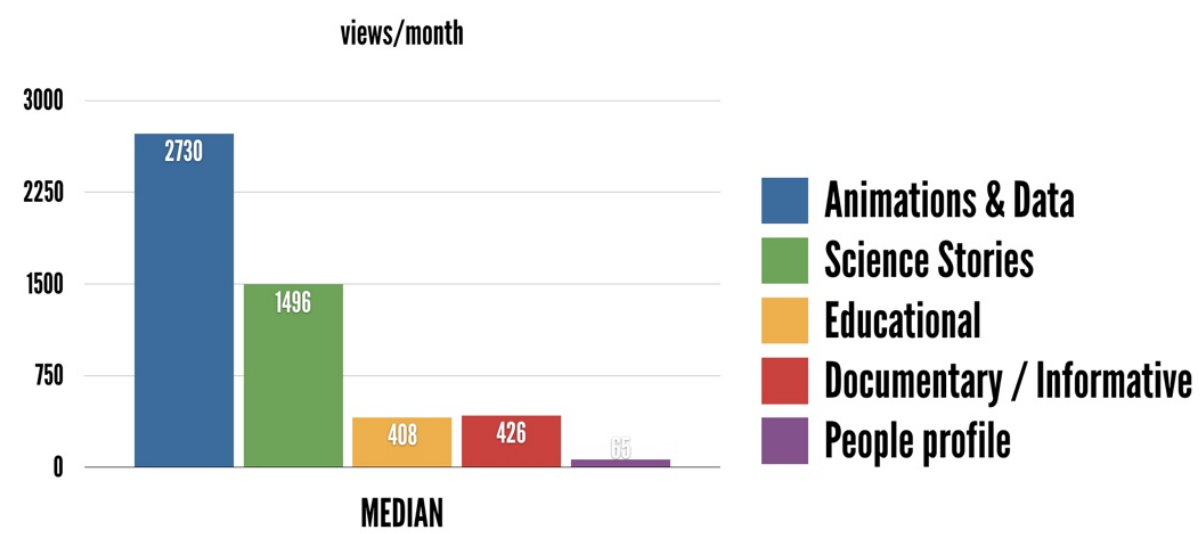

Fig. 2. The median value of the views per month for each category in the selection of 49 video of the NASA Explorer YouTube channel on 21 June 2017.

In Figure 3 bar diagram for the full date set for 21 June 2017 is shown.

\section{ESO, ESA AND NASA GSFC YOUTUBE CHANNEL; 106 VIDEOS; 21-06-2017}

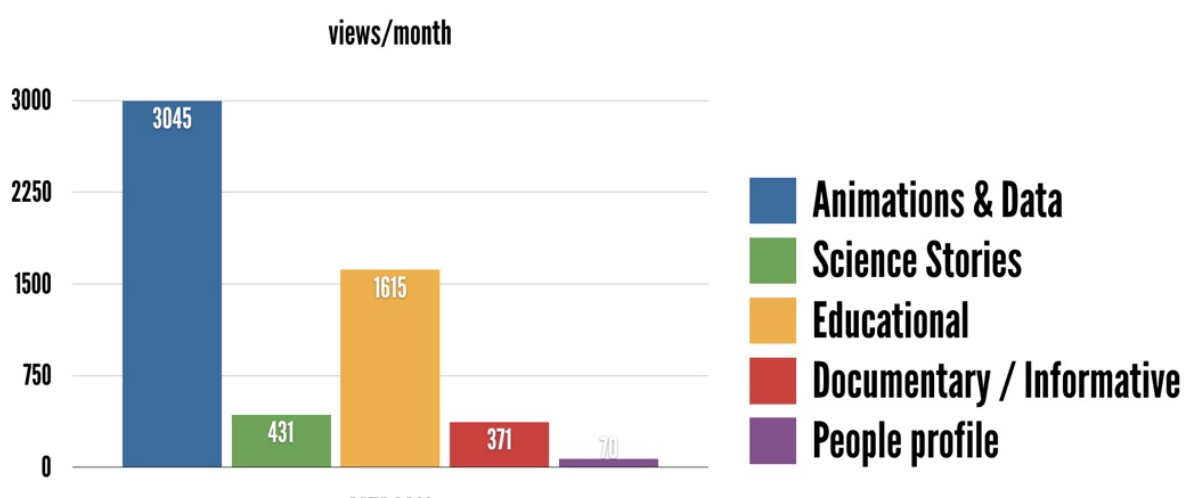

MEDIAN

Fig. 3. The median value of the views per month for each category in the full selection of 106 videos on 21 June 2017.

Clearly in all cases the Animations and Data category engages the largest YouTube audience by a factor of about two compared to the next most popular choice. This might seem surprising at first, but the explanation could be fairly straightforward. Most Animations and Data videos have a strong sound track, rather spectacular views, and often no explicit explanation in the form of a Voice Over or text. They have a high wow-factor, are easy to watch and, very importantly, play on the emotions of the viewer using strong music tracks. The importance of music cannot be underestimated: "if seeing is believing, 
then hearing is feeling" is a line used in the film industry, and indeed the music tells the viewer how to feel about what is being shown on the screen. These factors add to the entertainment value of the videos in this category.

The most popular video in this selection across all the categories is, no surprise, also from the Animations and Data category. It is a video found on the ESA YouTube channel, a time lapse from the International Space Station showing the Earth passing beneath, the day and night cycle and the amazing aurorae, of course accompanied by a mesmerising music track. The next most popular video is found on the NASA Explorer YouTube channel and shows an eruption on the Sun observed by SOHO, with a rather wild sound track.

\subsection{In more detail}

\subsubsection{Short fame}

A comparison between the bar diagrams in Figures 1 and 2 show that the relative popularity between the categories has moved very little over the four-year time-period between 15 April 2013 and 21 June 2017, but that the absolute number of views per month has decreased by roughly a factor three to four. In the last column of Table 1 the number of views on 21 June 2017 is expressed as a percentage of the projected number of views based on the results from the measurement on 15 April 2013. The median values in each category are on the order of $25-30 \%$ percent. It means that once a video has been posted, most of the views occur quite early after the posting date. Once this initial period is over little new views are added, and less and less as time goes on. This is in fact a well-known effect, as is shown by the example of the evolution of the daily views of one of the videos in Figure 4.

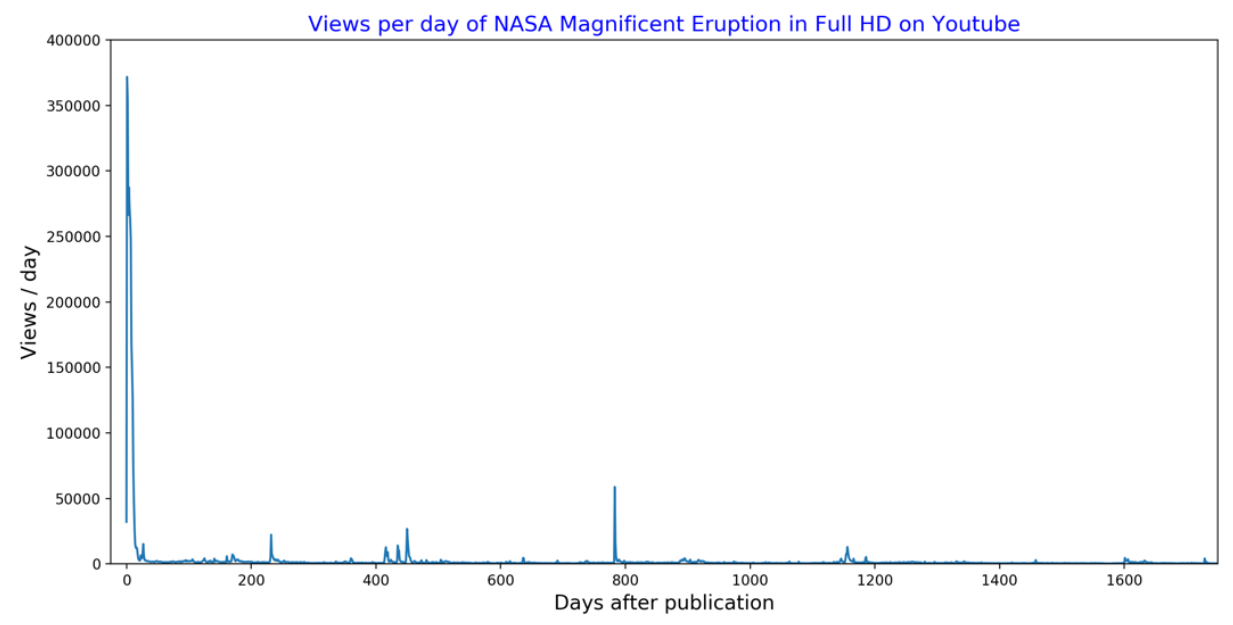

Fig. 4. The evolution of the number of views per day for the Magnificent Eruption in Full HD video on the NASA Explorer YouTube channel. 


\subsubsection{Publicity and actuality}

When adding the selected ESA and ESO videos to the NASA Explorer YouTube channel selection, the number of views in the education category increases by a factor of four, and the number of views in the Science Stories category decreases by a factor of three. From Table 2 it is seen that most of the selected educational videos for ESA are from the Rosetta mission. These are the most obvious educational videos on the ESA YouTube channel. These videos were extremely well received by the audience, and thus became very popular. They were also linked to specific events during the Rosetta mission, such as the wake up of Rosetta, the arrival, the landing of Philae, the end of the mission, and were extremely well publicised over social media, drawing a lot of attention.

The decrease in Science Stories is due to the unpopularity of the offered videos on both the ESA and the ESO YouTube channels. It is especially surprising to see the relatively low popularity of the ESO Hubble Cast series. Could it be explained by a lack of publicity?

\subsection{Value for money}

Finally, it is interesting to compare two videos from the full selection: the film "Ambition", the most popular in terms of views per month in the Educational category, and the "Alexander Gerst's Earth Time lapse" video, the most popular video in the Animation and Data category (Table 2). "Ambition" was published on 24 October 2014 and is 6m:41s long. "Alexander Gerst's Earth Time lapses" was published about two months later, on 22 December 2014 and is 6m:10s long.

"Ambition" is a very ambitious short film about the ESA Rosetta mission and the importance and search of water in the Solar System. It features actors Aidan Gillen, well known from the HBO series Games of Thrones, and Aisling Franciosi known from BBC series The Fall. Its date of publication was timed to be close to the Rosetta Philae landing, which occurred on 12 November 2014. As far as we know, the very high production value and associated high production costs are unheard of for this type of film produced by any space agency. We estimate the production cost to be on the order of 500,000 Euros. The number of views per month on 21 June 2017 for Ambition is 44,753. In our selection of videos, "Ambition" was the fourth most popular film in terms of number of views per month.

The most popular video, "Alexander Gerst's Earth Timelapses" in the Animation and Data category, has a much lower production cost, when of course not taking into account the cost of ESA astronaut Gerst's mission to the International Space Station. The cost to produce this video from the raw materials is on the order of 1,000 Euros and the number of views per month on 21 June 2017 is 97,710, about double the number for "Ambition".

Of course, the number of views per month is not the only factor that will determine the impact of a video and we do not want to imply that high production value standards should not be reached for. "Ambition" is very well produced and does provide an interesting experiment: clearly, the YouTube audience does not necessarily prefer this type over more "emotional" videos such as "Alexander Gerst's Earth Time lapses" or other similar videos. In the Animations and Data category there are three more videos from our selection that have higher views per months than "Ambition". 


\section{Concluding remarks}

This short paper is a first attempt to try to understand what type of videos with space themes are popular among the YouTube audience. The results seem to indicate that videos with a high entertainment value and little explicit explanation are preferred. This does not discredit any other video concept, because the number of views per month is not the only way to measure the impact of a video and its content. A video can have a small number of views (per month), but still have a high impact on a small niche audience targeted by the producers of the video.

Also, our study is by no means exhaustive. We plan to follow up this study by including more videos from the ESA, ESO and NASA websites, as well as other websites. Other parameters to be taken into account are the number of likes and shares, as well as the comments. Comments can help code for positive or negative emotions that a video provokes among the audience. Sharing of video content on other platforms, such as Facebook and Twitter are also important to take into account. Finally, social media is a rapidly changing environment. Our study spans four years between the first date of observation in April 2013 and the second in June 2017. During that time-period, the audience has changed: high school students went to in college, college students started to seek employment, etc. Each generation has its own interests, and more importantly, its own media use and preferences.

The evaluation of the reception and use of video content produces by space agencies should be a constant effort.

We do hope that this initial study can be a step in that direction and help video content producers at space organisations reach the target set by their mandates and understand the possible fate of their productions.

MR wishes to thank the organisers of the ISE2A for organising the conference and for accepting his contribution, as well as Robert Garner and Wade Sisler from NASA Goddard Space Flight Center for kindly providing detailed information on the views per day for the Magnificent Eruption in Full HD video for Figure 4.

\section{References}

1. European Space Agency YouTube channel URL: https://www.youtube.com/user/ESA

2. European Southern Observatory YouTube channel URL: https://www.youtube.com/user/ESOobservatory

3. NASA Goddard Space Flight Center YouTube channel URL: https://www.youtube.com/user/NASAexplorer 